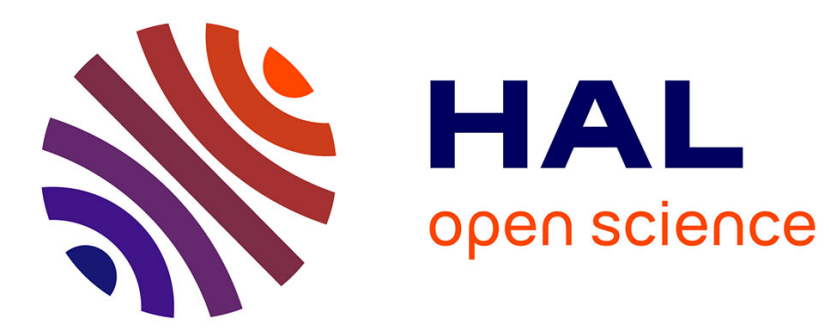

\title{
Élaboration et caractérisation de cristaux dérivés de K3Li2Nb5O15
}

Abdelwahed Zegzouti, A. Abalhassain, M. Elaatmani, Jean Ravez, Jean-Pierre Chaminade

\section{- To cite this version:}

Abdelwahed Zegzouti, A. Abalhassain, M. Elaatmani, Jean Ravez, Jean-Pierre Chaminade. Élaboration et caractérisation de cristaux dérivés de K3Li2Nb5O15. Journal de Physique III, 1996, 6 (6), pp.727-735. 10.1051/jp3:1996146 . jpa-00249487

\section{HAL Id: jpa-00249487 https://hal.science/jpa-00249487}

Submitted on 1 Jan 1996

HAL is a multi-disciplinary open access archive for the deposit and dissemination of scientific research documents, whether they are published or not. The documents may come from teaching and research institutions in France or abroad, or from public or private research centers.
L'archive ouverte pluridisciplinaire HAL, est destinée au dépôt et à la diffusion de documents scientifiques de niveau recherche, publiés ou non, émanant des établissements d'enseignement et de recherche français ou étrangers, des laboratoires publics ou privés. 


\title{
Élaboration et caractérisation de cristaux dérivés de $\mathrm{K}_{3} \mathrm{Li}_{2} \mathrm{Nb}_{5} \mathrm{O}_{15}$
}

A. Zegzouti $\left({ }^{1}\right)$, A. Abalhassain $\left({ }^{1}\right)$, M. Elaatmani $\left({ }^{1, *}\right)$, J. Ravez $\left({ }^{2}\right)$ et J.P. Chaminade $\left({ }^{2}\right)$

( $\left.{ }^{1}\right)$ Laboratoire de Chimie du Solide Minéral, Faculté des Sciences Semlalia,

Université Cadi Ayyad Marrakech, Maroc

(2) ICMCB,CNRS, Av. A. Schweitzer, 33608 Pessac, France

(Reçu le 20 septembre 1995, révisé le 9 janvier 1996, accepté le 26 février 1996)

PACS.77.80.-e - Ferroelectricity and antiferroelectricity

Résumé. - Des cristaux de bonne qualité de type $\mathrm{K}_{3} \mathrm{Li}_{2} \mathrm{Nb}_{5} \mathrm{O}_{15}$ et de structure "bronze" ont été élaborés par fusion et refroidissement lent en présence d'un pseudo-flux $\left(\mathrm{K}_{2} \mathrm{CO}_{3}\right.$ et $\mathrm{Nb}_{2} \mathrm{O}_{5}$ en excès). L'analyse chimique montre que leur composition correspond à la formulation $\mathrm{K}_{2,82}(\mathrm{Nb}-\mathrm{O})_{0,08} \mathrm{Li}_{1,54} \mathrm{Nb}_{5} \mathrm{O}_{15}$. Ces matériaux cristallisent dans le système quadratique à $295 \mathrm{~K}$ avec le groupe spatial $\mathrm{P} 4 \mathrm{bm}$. Les mesures diélectriques ont été effectuées selon deux directions [001] et [100], perpendiculaires aux grandes faces des cristaux élaborés. Les variations thermiques de la permittivité et de la biréfringence ont permis de mettre en évidence deux maxima l'un à $T_{1} \cong 100 \mathrm{~K}$ selon [100], l'autre à $T_{\mathrm{C}} \cong 680 \mathrm{~K}$ selon [001] ; ce dernier correspond à la température de Curie ferroélectrique.

\begin{abstract}
Good quality crystals of $\mathrm{K}_{3} \mathrm{Li}_{2} \mathrm{Nb}_{5} \mathrm{O}_{15}$ type were grown from the melt followed by method slow cooling using a pseudo-flux method (an excess of $\mathrm{K}_{2} \mathrm{CO}_{3}$ and $\mathrm{Nb}_{2} \mathrm{O}_{5}$ is present). The crystals were identified by X-ray diffraction, they crystallized with the tungsten bronzetype structure in the tetragonal system at room temperature. The space group is $\mathrm{P} 4 \mathrm{bm}$. Their composition $\left(\mathrm{K}_{2}{ }_{82}(\mathrm{Nb}-\mathrm{O})_{0}{ }_{08} \mathrm{Li}_{1.54} \mathrm{Nb}_{5} \mathrm{O}_{15}\right)$ was determined by chemical analysis. Dielectric measurements were realized along the [001] and [100] directions in the frequency range from $10^{3}$ to $3 \times 10^{5} \mathrm{~Hz}$. The temperature dependences of dielectric constants $\varepsilon_{\mathrm{r} 33}^{\prime}$ and $\varepsilon_{\mathrm{r} 11}^{\prime}$ and of birefringence $\Delta n$ show two maximums at $T_{1} \cong 100 \mathrm{~K}$ and $T_{\mathrm{C}} \cong 680 \mathrm{~K}$, the latter being the ferroelectric Curie temperature.
\end{abstract}

\section{Introduction}

Les cristaux ferroélectriques de type $\mathrm{K}_{3} \mathrm{Li}_{2} \mathrm{Nb}_{5} \mathrm{O}_{15}$ (KLN) et de structure bronze quadratique de tungstène présentent un grand intérêt en raison de leurs performances piézoélectriques, pyroélectriques et électro-optiques [1,2]. Leur croissance cristalline a fait l'objet de nombreux essais en utilisant plusieurs techniques de cristallogénèse afin d'obtenir des monocristaux de taille suffisante [3-7]. Des études diélectriques effectuées sur des monocristaux obtenus par la technique de Czochralski selon la direction [001] ont montré que les caractéristiques physiques et en particulier la température de Curie varient bien entendu avec la composition du bain : la valeur

${ }^{*}$ ) Auteur auquel doit être adressé la correspondance (Fax: (212) 4436769 ) 


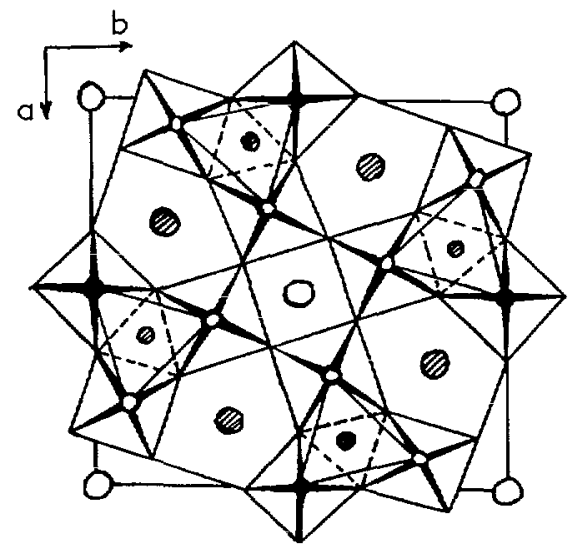
a)

$\begin{array}{cccccc}k & k & l i & -M b & M b \\ A & O & 0 & O & \bullet \\ A & B & C & M^{\prime} & M^{\prime \prime}\end{array}$

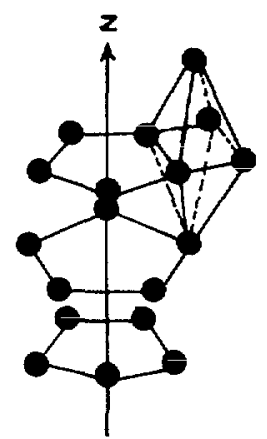

A

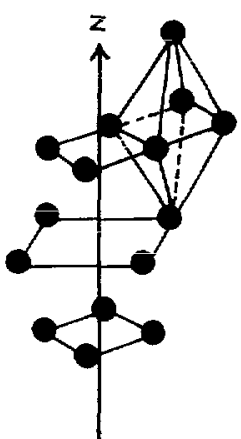

B

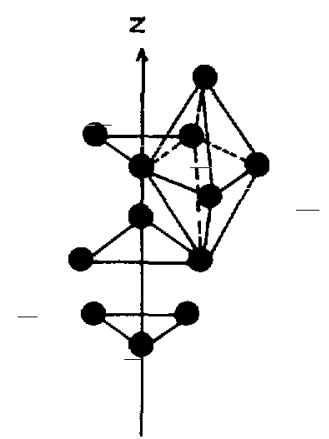

C

Fig. 1. - a) Projection du réseau des octaèdres sur le plan $x \mathrm{O} y$. b) Environnement oxygéné des tunnels anioniques.

[a) Projection of the cristalline network along $x \mathrm{O} y$. b) Anionic environments.]

de $T_{\mathrm{C}}$ décroît lorsque la quantité de niobium augmente [7]. La non-stcechiométrie est associée à un déficit de lithium et de potassium provenant de leur volatilisation sous forme $\mathrm{de} \mathrm{Li}_{2} \mathrm{O}$ et de $\mathrm{K}_{2} \mathrm{O}$, lors du chauffage. De même, d'autres études similaires sur céramiques de composition voisine de $\mathrm{K}_{3} \mathrm{Li}_{2} \mathrm{Nb}_{5} \mathrm{O}_{15}$ avaient montré l'existence de deux maxima, aux températures $T_{1}=105$ et $T_{\mathrm{C}}=705 \mathrm{~K}$, ce dernier correspond à la température de Curie ferroélectrique [8].

Nous nous sommes proposés dans le présent travail, d'élaborer des monocristaux de type (KLN) par la méthode de flux et de réaliser des études comparatives à celles effectuées antérieurement sur $\mathrm{K}_{3} \mathrm{Li}_{2} \mathrm{Nb}_{5} \mathrm{O}_{15}$ sous forme de céramiques [8]. Ces cristaux seront caractérisés par diffractométrie de RX, par analyse chimique et par des mesures de constantes diélectriques et de biréfringence optique.

La structure "bronze quadratique de tungstène" a été déterminée par Magneli en 1949 sur un monocristal de composition $\mathrm{K}_{0,57} \mathrm{WO}_{3}[9]$. Le réseau est constitué d'octaèdres $\mathrm{MO}_{6}$, légèrement distordus, liés entre eux par les sommets et laissant apparaître des cavités de type $A, B$ et $C$ 


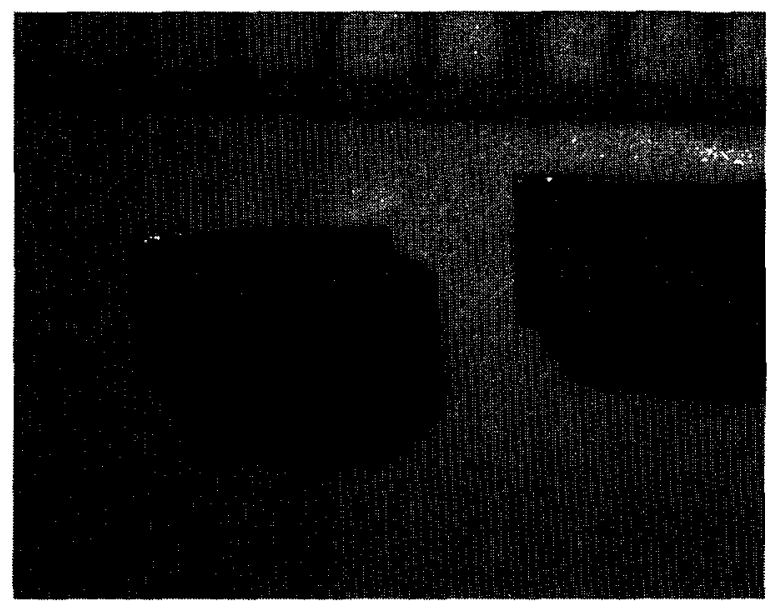

Fig. 2. - Photographie des cristaux obtenus.

[Photograph of the crystals.]

caractérisées respectivement par les coordinences 15, 12 et 9 (Fig. 1a). Juxtaposées les unes aux autres dans la direction $\mathrm{O} z$, ces cavités forment de véritables tunnels de section pentagonale, carrée ou triangulaire, où viennent s'insérer les ions métalliques (Fig. 1b).

Dans certains cas, ce sont des chaînons $\mathrm{M}-\mathrm{O}-\mathrm{M}$ dirigés suivant $\mathrm{O} z$ qui se logent uniquement dans les tunnels de large section (coordinences 15 ), en raison de la répulsion qui en résulterait entre ions oxygène des chaînons et ceux des octaèdres dans les autres sites [10]. Les atomes $M$ en coordinence octaédrique occupent deux types de sites : les sites $M^{\prime}$ en position générale et $\mathrm{M}^{\prime \prime}$ situés sur un axe binaire (Fig. 1a).

\section{Croissance cristalline}

Le diagramme de phase du système ternaire $\mathrm{K}_{2} \mathrm{O}-\mathrm{Li}_{2} \mathrm{O}-\mathrm{Nb}_{2} \mathrm{O}_{5}$ a été étudié antérieurement par Scott et Ikeda [7]. Ces derniers ont montré que la solution solide de type (KLN) possède un domaine très étendu au sein de ce système. Les cristaux sont préparés par la technique de flux (excès de $\mathrm{K}_{2} \mathrm{CO}_{3}$ et $\mathrm{Nb}_{2} \mathrm{O}_{5}$ ) à partir d'un mélange molaire de $35 \%$ de $\mathrm{K}_{2} \mathrm{CO}_{3}, 17,30 \%$ de $\mathrm{Li}_{2} \mathrm{CO}_{3}$ et $47,70 \%$ de $\mathrm{Nb}_{2} \mathrm{O}_{5}$ porté à $1150{ }^{\circ} \mathrm{C}$ pendant 4 heures, dans un creuset de platine. Après fusion, le produit est refroidi aux vitesses de $2^{\circ} \mathrm{C} /$ heure jusqu'à $850^{\circ} \mathrm{C}$, puis de $50^{\circ} \mathrm{C} /$ heure jusqu'à l'ambiante. Les cristaux ainsi obtenus sont transparents de couleur jaune verdâtre et se présentent en général sous deux formes, soit des plaquettes, soit des parallélépipèdes de dimensions maximales $4 \times 2 \times 0,8 \mathrm{~mm}$ (Fig. 2).

En tenant compte des résultats de l'analyse chimique effectuée sur ces cristaux et des considérations structurales indiquées ci-dessus, la composition du cristal est la suivante $\mathrm{K}_{2,82}(\mathrm{Nb}-\mathrm{O})_{0,08} \mathrm{Li}_{1,54} \mathrm{Nb}_{5} \mathrm{O}_{15}[10]$.

\begin{tabular}{|c|c|c|c|c|}
\hline élément & $\mathrm{O}$ & $\mathrm{K}$ & $\mathrm{Li}$ & $\mathrm{Nb}$ \\
\hline $\begin{array}{c}\% \text { massique } \\
\text { mesuré } \\
( \pm 0,02)\end{array}$ & 26,24 & 13,59 & 1,32 & 58,84 \\
\hline
\end{tabular}




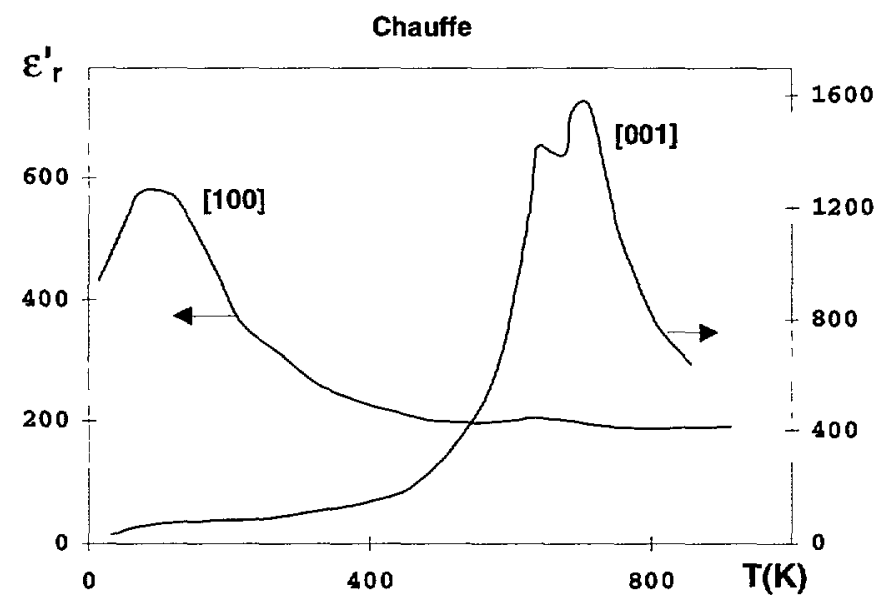

Fig. 3. - Variation thermique de $\varepsilon_{\mathbf{r}}^{\prime}$ en chauffe à $10^{3} \mathrm{~Hz}$ d'un monocristal de composition $\mathrm{K}_{2,82}(\mathrm{Nb}-\mathrm{O})_{0,08} \mathrm{Li}_{1,54} \mathrm{Nb}_{5} \mathrm{O}_{15}$ orienté suivant la direction [001] et [100].

[Thermal dependence of $\varepsilon_{r}^{\prime}$ on heating at $10^{3} \mathrm{~Hz}$ of a single crystal with composition $\mathrm{K}_{282}(\mathrm{Nb}-\mathrm{O})_{008} \mathrm{Li}_{154} \mathrm{Nb}_{5} \mathrm{O}_{15}$ oriented along [001] and [100].]

L'étude par les techniques classiques de diffractométrie de RX sur monocristal à l'ambiante (Laue, cristal tournant, Weissenberg) révèle que la symétrie est quadratique avec les paramètres cristallins suivants :

$$
\begin{aligned}
& a=12,520 \pm 0,001 \AA \\
& c=3,980 \pm 0,005 \AA \\
& \text { groupe d'espace : P4bm } \\
& Z=2 \\
& \text { densité : } 4,345 \pm 0,005 .
\end{aligned}
$$

\section{Mesures diélectriques}

Les variations thermiques de la constante diélectrique relative réelle $\varepsilon_{\mathbf{r}}^{\prime}$ et des pertes diélectriques tg $\delta$ ont été déterminées sur des plaquettes monocristallines de dimensions $4 \times 2 \times 0,8 \mathrm{~mm}$ et $3,5 \times 1,6 \times 0,8 \mathrm{~mm}$; les grandes faces sont perpendiculaires aux deux directions [100] et [001] respectivement. Des électrodes d'or sont déposées sur les faces des cristaux par l'intermédiaire d'une laque. Les mesures diélectriques sont effectuées à basse et haute températures de 4 à $300 \mathrm{~K}$, à l'aide d'un pont de capacité automatique WAYNE-IKERR B905, aux fréquences $10^{2}, 10^{3}$ et $10^{4} \mathrm{~Hz}$. Les mesures de 300 à $900 \mathrm{~K}$ sous hélium sec sont réalisées en utilisant un analyseur de type WAYNE-KERR modèle 6425 à diverses fréquences $\left(10^{3}, 1,5 \times 10^{3}, 3 \times 10^{3}\right.$, $10^{4}, 3 \times 10^{4}, 10^{5}$ et $3 \times 10^{5} \mathrm{~Hz}$ ). La figure 3 montre les variations thermiques de la permittivité $\varepsilon_{\mathrm{r}}^{\prime}$ à $10^{3} \mathrm{~Hz}$ selon [001] et [100]. Il apparaît trois maxima réversibles selon la direction [001]. Le premier, de très faible intensité est observé à $T_{1} \cong 100 \mathrm{~K}$; le deuxième et le troisième, d intensités élevées, sont relativement proches à $T_{2}=635 \pm 10$ et $T_{3}=680 \pm 10 \mathrm{~K}$. En revanche, selon la direction [100] il n'y a que deux pics, le premier à $T_{1}=100 \mathrm{~K}$ étant nettement supérieur en intensité au second à $T_{3} \cong 650 \mathrm{~K}$.

En vue de déterminer l'origine des deux pics à 635 et $680 \mathrm{~K}$, une étude en fréquence a été réalisée. Les figures 4,5 et 6 illustrent de 300 à $900 \mathrm{~K}$ les évolutions thermiques de $\varepsilon_{\mathrm{r}}^{\prime}$ et de $\operatorname{tg} \delta$ en montée ou en descente de température réalisées à différentes fréquences suivant la 


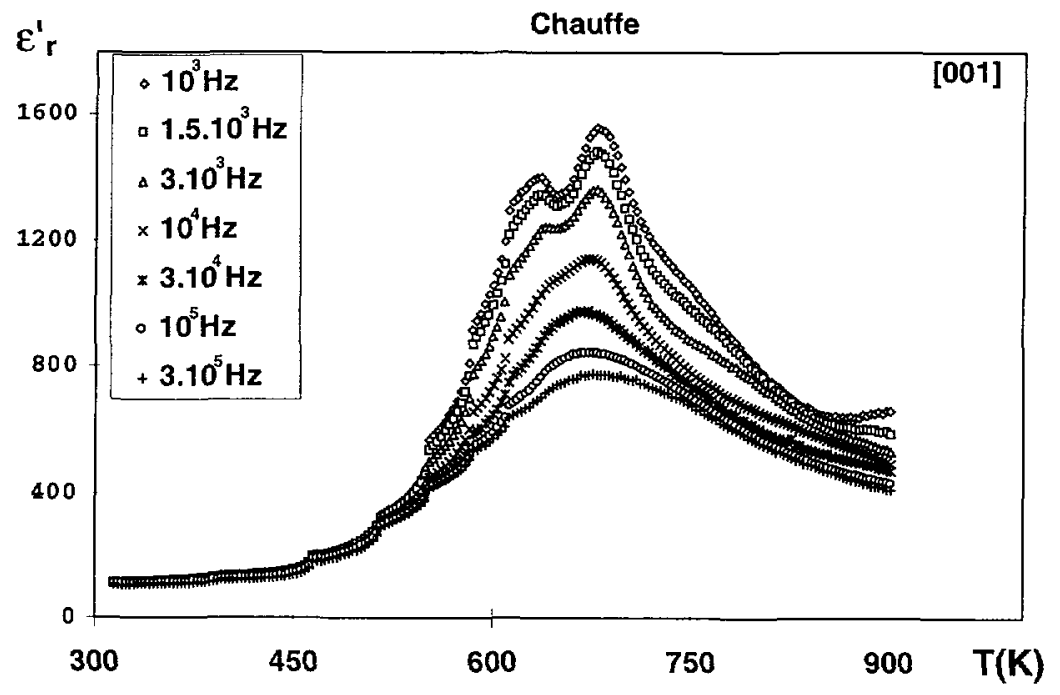

Fig. 4. - Variation thermique de $\varepsilon_{\mathrm{r}}^{\prime}$ en chauffe à diverses fréquences d'un monocristal de composition $\mathrm{K}_{2,82}(\mathrm{Nb}-\mathrm{O})_{0,08} \mathrm{Li}_{1,54} \mathrm{Nb}_{5} \mathrm{O}_{15}$ orienté suivant la direction [001].

[Thermal dependence of $\varepsilon_{\mathrm{r}}^{\prime}$ on heating at various frequencies of a single crystal with composition $\mathrm{K}_{282}(\mathrm{Nb}-\mathrm{O})_{0}{ }_{08} \mathrm{Li}_{1.54} \mathrm{Nb}_{5} \mathrm{O}_{15}$ oriented along [001].]

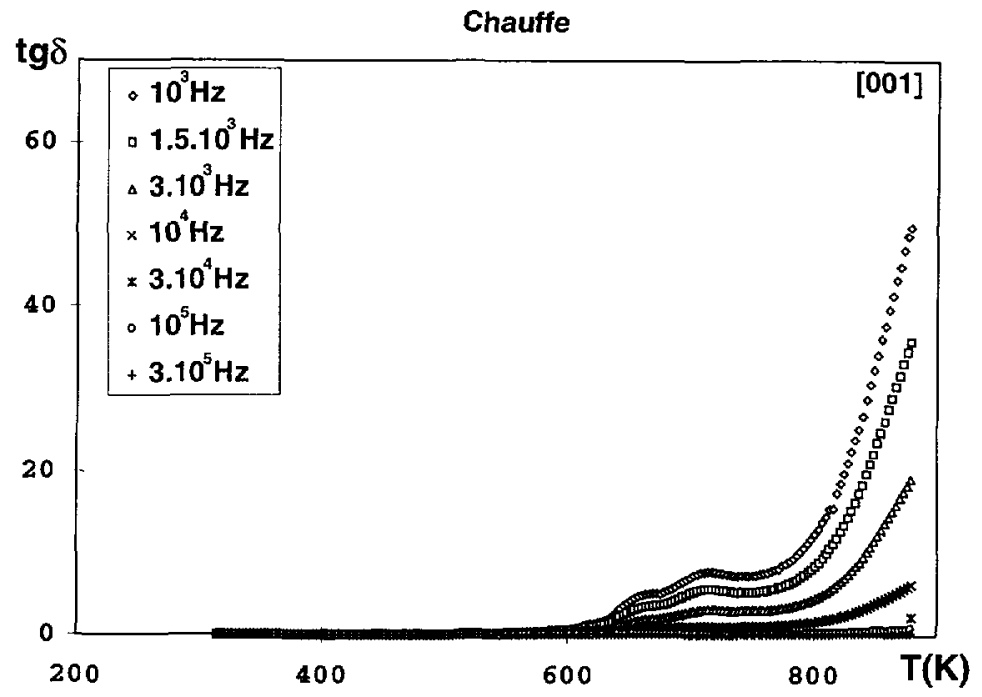

Fig. 5. - Variation thermique de tg $\delta$ en chauffe à diverses fréquences d'un monocristal de composition $\mathrm{K}_{2,82}(\mathrm{Nb}-\mathrm{O})_{0,08} \mathrm{Li}_{1,54} \mathrm{Nb}_{5} \mathrm{O}_{15}$ orienté suivant la direction [001].

[Thermal dependence of $\operatorname{tg} \delta$ on heating at various frequencies of a single crystal with composition $\mathrm{K}_{2}{ }_{82}(\mathrm{Nb}-\mathrm{O})_{0}{ }_{08} \mathrm{Li}_{1}{ }_{54} \mathrm{Nb}_{5} \mathrm{O}_{15}$ oriented along [001].] 


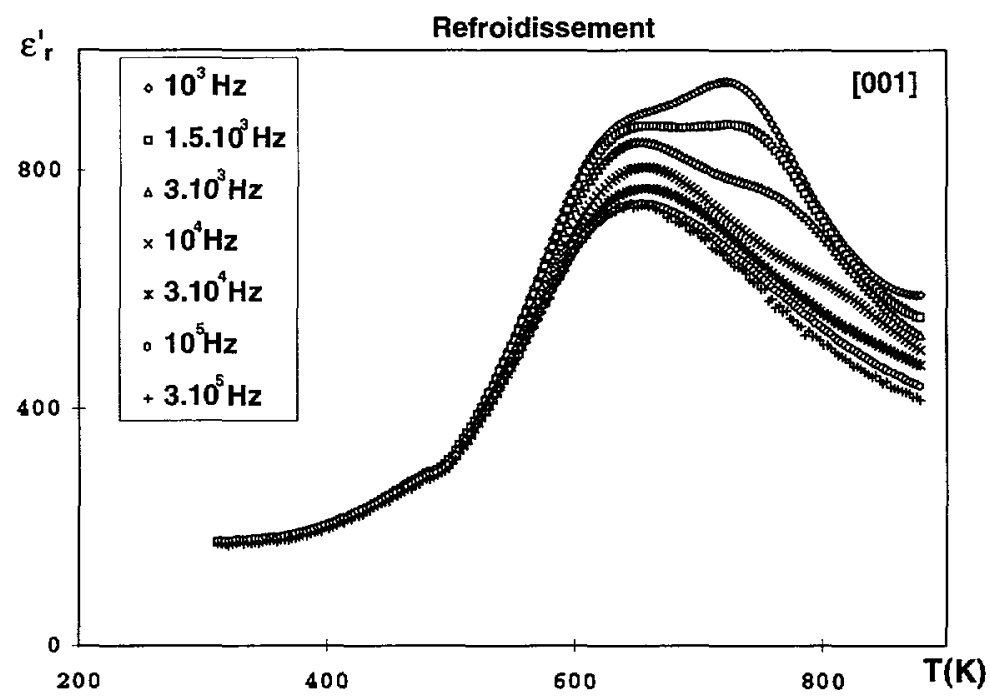

Fig. 6. - Variation thermique de $\varepsilon_{\mathrm{r}}^{\prime}$ au refroidissement à diverses fréquences d'un monocristal de composition $\mathrm{K}_{2,82}(\mathrm{Nb}-\mathrm{O})_{0,08} \mathrm{Li}_{1,54} \mathrm{Nb}_{5} \mathrm{O}_{15}$ orienté suivant la direction [001].

[Thermal dependence of $\varepsilon_{\mathrm{r}}^{\prime}$ on cooling at various frequencies of a single crystal with composition $\mathrm{K}_{2}{ }_{82}(\mathrm{Nb}-\mathrm{O})_{0}{ }_{08} \mathrm{Li}_{1}{ }_{54} \mathrm{Nb}_{5} \mathrm{O}_{15}$ oriented along [001].]

direction [001]. Les deux maxima sont observés aux basses fréquences à $T_{2}$ et $T_{3}$. Quand la fréquence augmente, le premier pic à $T_{2}$ évolue avec la fréquence, en particulier sa température augmente. Le second à $T_{3}$ ne varie pratiquement pas en température quelle que soit la valeur de la fréquence. La transition à $T_{3}$ est diffuse. En effet, d'une part la largeur à mi-hauteur est grande, d'autre part la température correspondant au minimum de $\operatorname{tg} \delta$ ne cö̈ncide pas avec la température du maximum de la permittivité relative réelle $[11,12]$. Les figures 4 et 6 montrent par ailleurs que la transition à $T_{3}$ présente une forte hystérésis thermique caractéristique d'une transition du premier ordre.

\section{Mesures optiques}

L'étude optique a été effectuée à l'aide d'un microscope polarisant type LEITZ-ORTHOLUX II POL en lumière blanche. Le cristal choisi est une plaquette cristalline à faces parallèles orientée perpendiculairement à l'axe optique, d'indice de réfraction $n=1,77 \pm 0,005$ et d'épaisseur $70 \mu \mathrm{m}$. L'étude des variations thermiques de la biréfringence en montée et en descente de température a été effectuée à l'aide d'un compensateur de type BEREK en lumière polarisée $\lambda=543 \mathrm{~nm}$. Ces mesures sont réalisées soit sous courant d'azote sec grâce à l'utilisation d'une platine chauffante Leitz pour les hautes températures (300 à $900 \mathrm{~K}$ ), soit sous courant d'azote sec refroidi par passage dans l'azote liquide, dans une cellule basse température en verre pour des observations au dessous de la température ambiante $(77$ à $300 \mathrm{~K})$. La figure 7 montre l'existence d'une discontinuité à la température $680 \pm 10 \mathrm{~K}$. Une observation en lumière polarisée permet de déterminer le signe optique du matériau : celui-ci est uniaxe négatif à $300 \mathrm{~K}$. 


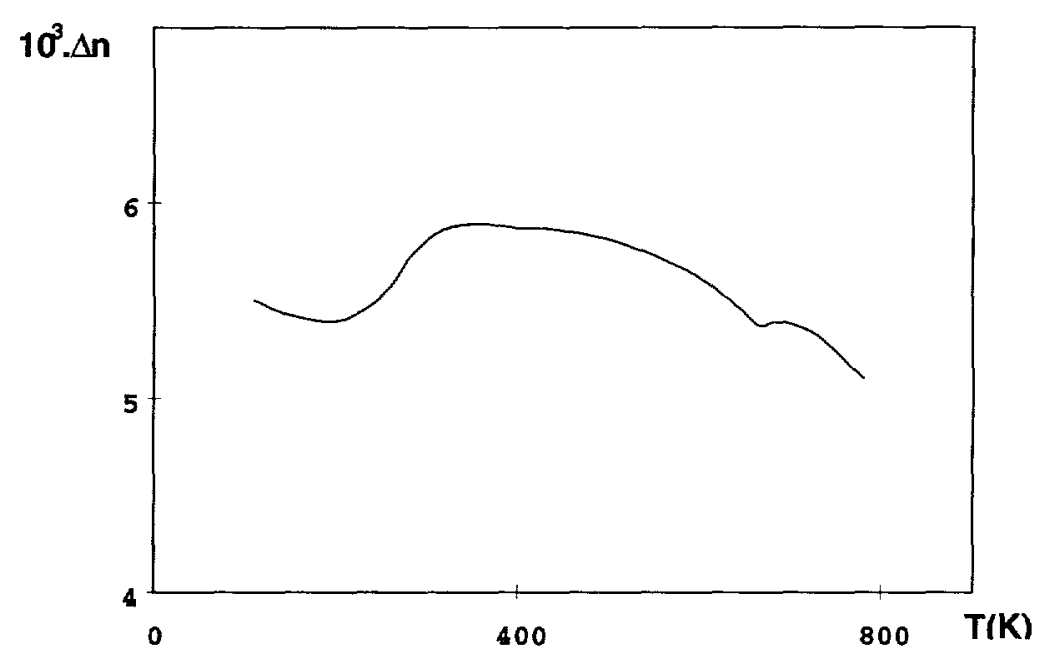

Fig. 7. - Variation thermique de la biréfringence $\Delta n$ en chauffe d'un monocristal de composition $\mathrm{K}_{2,82}(\mathrm{Nb}-\mathrm{O})_{0,08} \mathrm{Li}_{1,54} \mathrm{Nb}_{5} \mathrm{O}_{15}$ orienté perpendiculairement à la direction [001].

[Thermal dependence of the birefrigence $\Delta n$ of a single crystal with composition $K_{2.82}$ $(\mathrm{Nb}-\mathrm{O})_{0.08} \mathrm{Li}_{1.54} \mathrm{Nb}_{5} \mathrm{O}_{15}$ oriented perpendicularly to the [001] direction.]

\section{Résultats et discussion}

Des cristaux de structure bronze et dérivés de $\mathrm{K}_{3} \mathrm{Li}_{2} \mathrm{Nb}_{5} \mathrm{O}_{15}$ ont été préparés par fusion et refroidissement lent en présence d'un pseudo-flux $\left(\mathrm{K}_{2} \mathrm{CO}_{3}\right.$ et $\mathrm{Nb}_{2} \mathrm{O}_{5}$ en excès). Ce procédé a permis d'isoler des monocristaux dont la taille s'est avérée suffisante pour la caractérisation des propriétés physiques. L'analyse chimique montre que leur composition correspond à la formulation $\mathrm{K}_{2,82}(\mathrm{Nb}-\mathrm{O})_{0,08} \mathrm{Li}_{1,54} \mathrm{Nb}_{5} \mathrm{O}_{15}$. L'étude par diffraction $\mathrm{X}$ à $295 \mathrm{~K}$ révèle que les cristaux obtenus cristallisent dans le système quadratique avec le même groupe d'espace et le même nombre de motifs par maille que $\mathrm{K}_{3} \mathrm{Li}_{2} \mathrm{Nb}_{5} \mathrm{O}_{15}$ [13]. Les mesures diélectriques ont été effectuées à basse et haute températures selon [100] et [001]. Un maximum de permittivité a été observé à la température $T_{1}=100 \mathrm{~K}$ selon [100]. Les mesures diélectriques selon [001] réalisées à diverses fréquences et dans la gamme de température de 300 à $900 \mathrm{~K}$, montrent deux pics intenses à basse fréquence aux températures $T_{2}=635 \mathrm{~K}$ et $T_{3}=680 \mathrm{~K}$. Quand la fréquence augmente, le premier pic à $T_{2}$ diminue en intensité et se déplace vers les hautes températures impliquant ainsi une relaxation diélectrique ; ce phénomène est dû probablement à une conductivité ionique en relation avec la mobilité des ions $\mathrm{Li}^{+}$dans les cavités de type $\mathrm{C}$ et parallèlement à l'axe $\mathrm{O} z$. La température $T_{3}$ du second pic est indépendante de la valeur de la fréquence. Ce résultat ajouté à l'anomalie de $\Delta n$ observée à la même température implique que $T_{3}$ correspond à la température de Curie $T_{\mathrm{C}}$ ferroélectrique. L'ordre de grandeur de la valeur de $T_{\mathrm{C}}$ pour $\mathrm{K}_{3} \mathrm{Li}_{2} \mathrm{Nb}_{5} \mathrm{O}_{15}\left(T_{\mathrm{C}}=683 \mathrm{~K}\right)$ est d'ailleurs un argument supplémentaire [10]. La comparaison des intensités des pics observés à $T_{C}$ selon [100] et [001] laisse penser que la polarisation spontanée est bien dirigée suivant l'axe $\mathrm{O} z$. Ce résultat est en accord avec les travaux antérieurs relatifs aux mesures diélectriques ayant été effectuées uniquement selon [001] $[4,7]$. Par ailleurs la quantité de niobium donnée par l'analyse chimique permet de déterminer la valeur de $T_{C}$ en s'appuyant sur les courbes de $T_{C}$ en fonction de taux du niobium donné antérieurement [7]; la valeur trouvée $\left(T_{\mathrm{C}}=685 \pm 5 \mathrm{~K}\right)$ est en bon accord avec celle donnée par 
mesures diélectriques $T_{\mathrm{C}}=680 \pm 10 \mathrm{~K}$. Les pics faibles observés selon [100] à $T_{\mathrm{C}}$ et selon [001] à $T_{1}$ proviennent vraisemblablement d'un petit écart d'orientation de ces deux cristaux. L'état diffus de la transition ferroélectrique-paraélectrique a fait l'objet de nombreuses recherches ; plusieurs mécanismes ont été suggérés [14]. La non-stœchiométrie, impliquant un désordre structural probable dans les sites A,B et C, contribue ici à l'état diffus de la transition [4]. Les deux maxima observés à 100 et $680 \mathrm{~K}$ confirment une étude réalisée sur une céramique de composition voisine [8].

Une étude est en cours pour caractériser la relaxation diélectrique, au voisinage de $T_{\mathrm{C}}$. Ce travail se situe dans le cadre plus large d'une étude systématique des relations fréquence de relaxation-température de Curie-liaison chimiques dans des matériaux ferroélectriques de type "bronze" en particulier.

\section{Bibliographie}

[1] Geusic J.E., Levinstein H.J., Rubin J.J., Singh S. et van Uitert L.G., The nonlinear optical properties of $\mathrm{Ba}_{2} \mathrm{NaNb}_{5} \mathrm{O}_{15}$, Appl. Phys. Lett. 11 (1967) 269.

[2] Ouwerkerk M., Potassium lithium niobate - a frequency doubler for (Al,Ga) as lasers, $A d v$. Materials 3 (1991) 399.

[3] Adachi M., Ph. D. Thesis, Kyoto University, Kyoto, Japan (1982).

[4] Jin B.M., Bhalla A.S., Choi Byung-Chun et Kim Jung-Nam, Dielectric anomalies in $\mathrm{Li}_{0.4} \mathrm{~K}_{0.6} \mathrm{NbO}_{3}$ crystals, Phys. Stat. Sol. 140 (1993) 239.

[5] Fukuda T., Growth and crystallographic characteristics of $\mathrm{K}_{3} \mathrm{Li}_{2} \mathrm{Nb}_{5} \mathrm{O}_{15}$ single crystals, Jpn. J. Appl. Phys. 8 (1969) 122.

[6] Wiegel M., Blasse G. et Ouwerkerk M., Luminescence of potassium lithium niobate compositions, Mat. Res. Bull. 27 (1992) 617.

[7] Scott B.A., Giess E.A., Olson B.L., Burns G., Smith A.W. et O'Kane D.F., The tungsten bronze field in the system $\mathrm{K}_{2} \mathrm{O}-\mathrm{Li}_{2} \mathrm{O}-\mathrm{Nb}_{2} \mathrm{O}_{5}$, Mat. Res. Bul. 5 (1970) 47.

[8] Elaatmani M., Zegzouti A., Ravez J. et Hagenmuller P., Nouvelles familles de matériaux à couplage ferroélastique-ferroélectrique de type "bronze" contenant simultanément le plomb et le lithium, Rev. Chim. Minér. 23 (1986) 290.

[9] Magneli A., Studies on the hexagonal tungsten bronzes of potassium rubidium and cesium, Acta Chem. Scand. 7 (1953) 315.

[10] Ravez J., Perron-Simon A. et Hagenmuller P., Les phases de structure "bronzes de tungstène quadratiques" - règles cristallochimiques, relations entre propriétés ferroélectriques et distortions structurales, Ann. Chım. tome 1 (1976) 251.

[11] Lines M.E. et Glass A.M., Principles and applications of ferroelectrics and related materials, Oxford University Press, London (1982) p. 286.

[12] Maeda K., Tasaka A. et Inagaki N., Ferroelectric behavior of vinylidenefluoridetrifluoroethylene-hexafluoropropylene terpolymers, Jpn. J. Appl. Phys. 30 (1991) 716.

[13] Abrahams S.C., Jamieson P.B. et Bernstein J.L, Ferroelectric tungsten bronze-type crystal structures. III. Potassium lithium niobate $\mathrm{K}_{(6-x-y)} \mathrm{Li}_{(4+x)} \mathrm{Nb}_{(10+y)} \mathrm{O}_{30}$, J. Chem. Phys. 54 (1971) 2355.

[14] Setter N. et Cross L.E., The Contribution of structural disorder to diffuse phase transition in ferroelectris, J. Mat. Sci. 15 (1980) 247. 